\title{
Automated Image Analysis of Iron-Ore Pellet Structure using Optical Microscopy
}

\author{
F. Nellros ${ }^{\mathrm{a}, *}$, M.J. Thurley ${ }^{\mathrm{a}, * *}$ \\ ${ }^{a}$ Luleå University of Technology, Dep. of Computer Science, Electrical \& Space \\ Engineering, Luleå SE-97187, SWEDEN
}

\begin{abstract}
Knowledge about pellet microstructure such as porosity and oxidation degree is essential in improving the pellet macro behavior such as structural integrity and reduction properties. Manual optical microscopy is commonly used to find such information but is both highly time consuming and operator dependent. This paper presents research to automate image capture and analysis of entire cross-sections of baked iron ore pellets to characterize proportions of magnetite, hematite, and other components.

The presented results cover: semi-automated image acquisition of entire pellets, separation of pellet and epoxy and calculation of total percentages of magnetite, hematite and pores. Using the Leica Qwin microscope software and a segmentation method based on Otsu thresholding these three objectives have been achieved with the phases labelled as magnetite, hematite and pores \& additives. Furthermore, spatial distributions of magnetite, hematite and pores \& additives are produced for each pellet, graphing proportions in relation to the distance to the pellet surface. The results are not directly comparable to a chemical analysis but comparisons with manual segmentation of images validates the method. Different types of pellets have been tested and the system has produced robust results for varying cases.
\end{abstract}

\section{Introduction}

In the mining of iron ore, refining and concentration is performed to produce a high grade fine iron ore product. At LKAB this concentrate is pelletized to produce their main product, balled iron ore pellets for either blast furnace or direct reduction steel production. LKAB mines are among the very few iron ore mines in the world that predominantly use magnetite instead of hematite ore resulting in specialized production conditions (Niiniskorpi, 2004).

Knowledge of the pellet microstructure is essential in improving the quality of the product. Data from microscopy is vital to understand what happens within the pellets on a micro-scale, which in turn affects the macro-behavior. This data can be used to understand current product conditions and as a reference

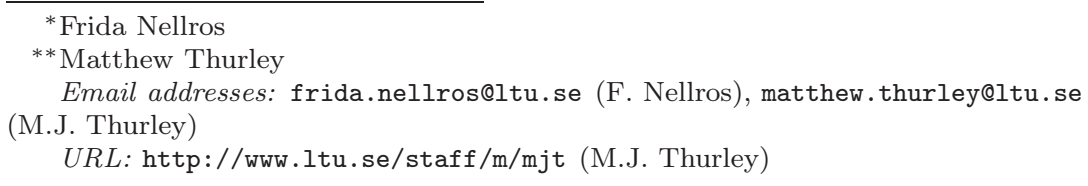


for possible future deviations. It is also important to understand what happens with the product when doing deliberate process changes as a part of product development.

Manual optical microscopy can be used to find such information but the results vary a lot with the expertise of the observer. Furthermore it is impractical to manually get a quantitative repeatable measurement of an entire pellet even using an objective magnification as low as 10 times (at least 100 images or more). This has initiated many attempts to automate some or all of the measurement and analysis steps.

In this paper the following literature review is restricted to research that both describes automated image analysis of optical microscopy and detail the methods that are used to achieve these results. Scanning Electron Microscopy (SEMs) are inappropriate for classifying iron oxides and are therefore not included in this review.

A number of studies have to varying degrees automated image capture in optical microscopy of iron ore (Lessa et al., 2007; Alvarez et al., 2008; Gomes and Paciornik, 2008; Wagner et al., 2008; Nellros, 2010) but few have developed fully automated image analysis techniques. The majority of techniques are semi-automated and apply an expert user to define how the image histogram should be classified into phases and then this classification is used on all subsequent images (Lessa et al., 2007; Alvarez et al., 2008; Wagner et al., 2008; Gomes and Paciornik, 2008). The risk of such approaches is that manually specified settings are dependent on opinion, and not necessarily robust to variation in image data over time such as from degradation in microscope illumination.

Investigation of colored light and polarized light imaging of various iron bearing minerals (Pirard and Lebichot, 2004; Pirard et al., 2007) has been performed. A stack of colored and polarized light images from a single measurement site are shown and classified (Pirard and Lebichot, 2004) into hematite, magnetite, pores and various other types of iron bearing minerals. The results require five images with different colored light and four images with different polarization angles making it an impractical image capture task if one done not have the specialized imaging system used by (Pirard and Lebichot, 2004).

In the presented work, semi-automated image capture of entire pellets is performed at 20 times resolution by visible light reflectance microscopy, collecting and integrating over 400 images per pellet. Additionally, fully automated image segmentation of the magnetite, hematite, surrounding epoxy, and other material (encompassing pores and additives), is performed.

\section{Method}

\subsection{Image acquisition}

Sample pellets are cast into epoxy, vacuum impregnated, cut in half and polished to give a good, reflecting cross section to study with the microscope. Leica Qwin software modules have been adapted through QUIPS programming using the provided Leica Qwin interface to obtain a routine that semi-automatically acquires images of an entire pellet. The approach has been to base the routine on the applet Mosaic included in the QGallery toolbox since it calculates the desired continuous scanning pattern.

The user inputs coordinates for the left and upper edges of the measurement area together with the size of the measurement area in millimeters and the 
height interval for automatic focus. The motorized stage then performs step-wise displacements in a zig-zag pattern from the upper left corner to the lower right as illustrated in figure 1. Images of 1088x816 pixels are retrieved at each stage position and a defocused reference image is automatically subtracted to remove uneven illumination effects (Ray, 1994, vignetting correction) before saving. Initialization of the image retrieval is thus manual for each pellet, however the sequential image retrieval is unsupervised.

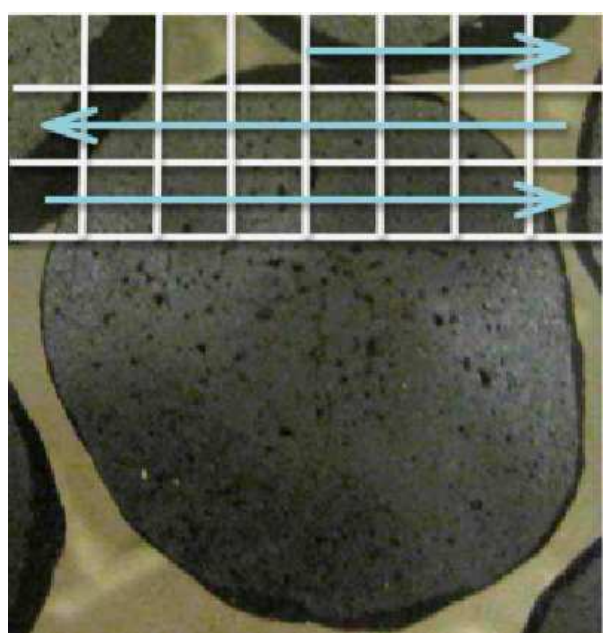

Figure 1: Principle of the scanning superimposed on one cut and polished pellet cast into epoxy.

\subsection{Image processing}

The following image processing strategies apply some mathematical morphology techniques. These techniques are particularly useful for identifying spatial structure in image data. Morphological image processing operations typically apply a structuring element, which is a spatial probe similar to a neighborhood operation, to the image. The reader is referred to the text Hands-On Morphological Image Processing (Dougherty and Lotufo, 2003) for a very clear explanation of this topic, or to any other good text on image processing if they require more information on these morphological image processing operations.

The image processing is performed in MATLAB (MATLAB, 2010) using the image processing toolbox and is divided into the following 3 parts;

1. identification of pellet area,

2. finding global information,

3. segmentation of individual images.

\subsubsection{Identification of pellet area}

The images from the pellet scan can be of four different types;

1. all pellet structure,

2. all epoxy, 
3. border image including pellet edge and surrounding epoxy and/or neighboring pellet,

4. pellet structure from a neighboring pellet.

The pellet area is found by analyzing an overview mosaic image as shown in figure 2a. This mosaic is created by simply connecting all the individual microscope images together in the order they were captured after downsampling them to one eighth of their original area.

The areas in the mosaic image containing pellet are identified using the following image processing strategy, described below in text and in algorithm 2.1 (page 6) in pseudo-code;

1. perform an edge detection algorithm on the overview mosaic (figure 2a to create the image shown in figure 2b, This is a grayscale image where sharp edges, i.e. rapid color transitions, has the highest values. The chosen edge detection is a morphological gradient (Dougherty and Lotufo, 2003) using a 3 pixel radius disk structuring element).

2. threshold the gradient image into a binary image using Otsu's method (Otsu, 1979) to single out the most distinct edges.

The detected and thresholded edges will occur at grain boundaries so the result is a binary image where white regions indicate the areas containing pellet. As can be seen in figure $2 \mathrm{~b}$ these regions will contain many holes that need to be filled to create a solid binary representation of the entire pellet region.

3. smooth edges and fill small holes of the binary edge image using a morphological closing with an 8 pixel radius disk structuring element

4. fill the remaining holes in the pellet areas using the MATLAB function imfill Dougherty and Lotufo, 2003, 3.5 Marker Selection in Reconstruction),

5. shrink the pellet area to compensate for step 3 so that it more accurately corresponds to the actual border of the pellet. The method used is a morphological erosion with a radius 4 disk-shaped structuring element, which can be visually interpreted as peeling off a 4 pixel wide layer of the binary pellet region.

The final result of this strategy is shown in figure 3a where white areas correspond to pellet. We see that there are parts of neighboring pellets in the mosaic image that need to be removed so we can identify only the sub-images that correspond to the pellet we are analyzing. In a less complicated situation this could have been done by simply removing white regions connected to the boundary of the binary mosaic image but there are some special cases where this is not possible. These two cases are highlighted by figure 3a where the pellet of interest both is touching other pellets in the sample and has not been entirely imaged, and is therefore touching the boundary of the mosaic image.

We assume that the center of the mosaic image always belongs to the pellet of interest, and apply the following strategy to remove all the components of other pellets present in the mosaic image. 

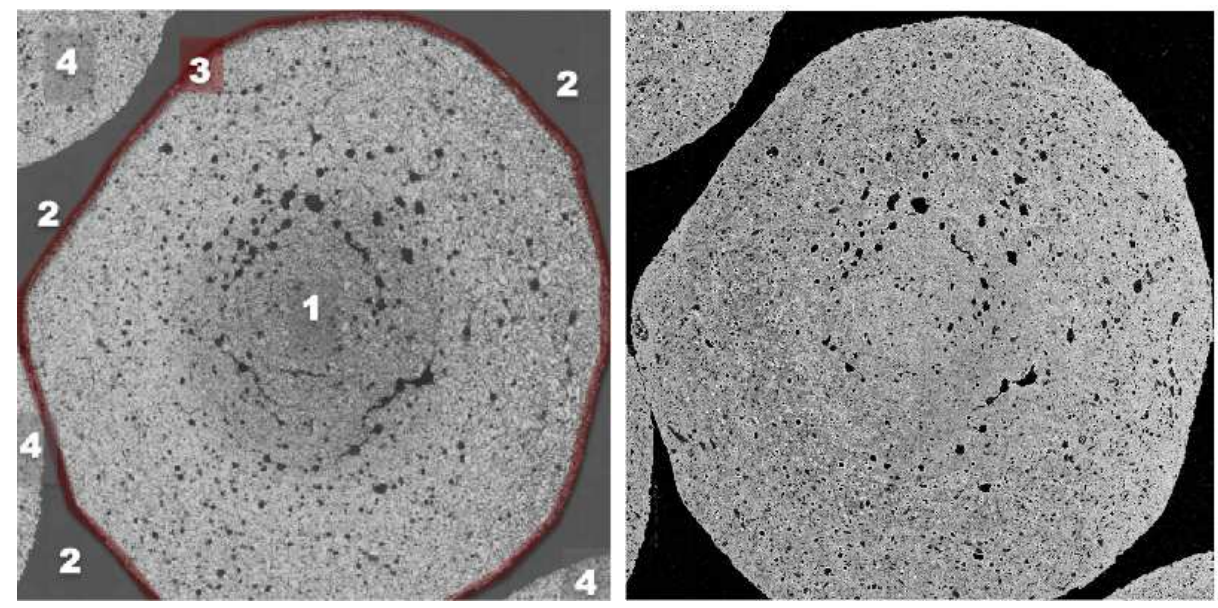

(a) Regions for different image types marked on(b) Morphological gradient of the grayscale iman overview mosaic.

age in (a)

Figure 2: Overview mosaic and its corresponding morphological gradient.

1. identify connected regions in image 3a using a region labeling technique (Dougherty and Lotufo, 2003, 3.3 Reconstruction), in this case the MAT$\mathrm{LAB}$ function bwlabel (three distinct connected regions are shown in figure 3a).

2. for each region $i$, check if the centre of the image is contained within it. If not, then subtract that region from the image and save it as a marker region $M_{i}$ to use in the segmentation in step 6

3. shrink the remaining pellet areas (white areas) by performing a morphological erosion (using a 2 pixel radius disk structuring element), which again can be visually interpreted as peeling off a 2 pixel wide layer along the outer perimeter of the binary regions.

Each time we do this erosion will make the pellet areas smaller, and will eventually separate connected pellets and could also entirely remove parts of pellets connected to the boundary.

4. test if the centre of the image is connected to the boundary by comparing the labelled number of the center pixel to the region numbers present at the image boundary. If so repeat from step 1 .

5. when the middle is no longer boundary connected, save the remaining region as the last marker region.

6. perform binary image segmentation on figure $3 \mathrm{a}$ using the distance transform and watershed segmentation based on the set of marker regions $M$. This is a well known segmentation algorithm documented in many sources (Beucher and Mever, 1992; Dougherty and Lotufo, 2003) and in the documentation for the MATLAB function watershed. The marker regions identified in the previous steps act as seeds that initialize each segmented region which are then sequentially grown in the watershed process until all parts of the input image belong to a segmented region. 
7. The resultant watershed segmentation separates connected pellets into individual regions. Extracting the region containing the image centre provides the image shown in figure $3 \mathrm{~b}$

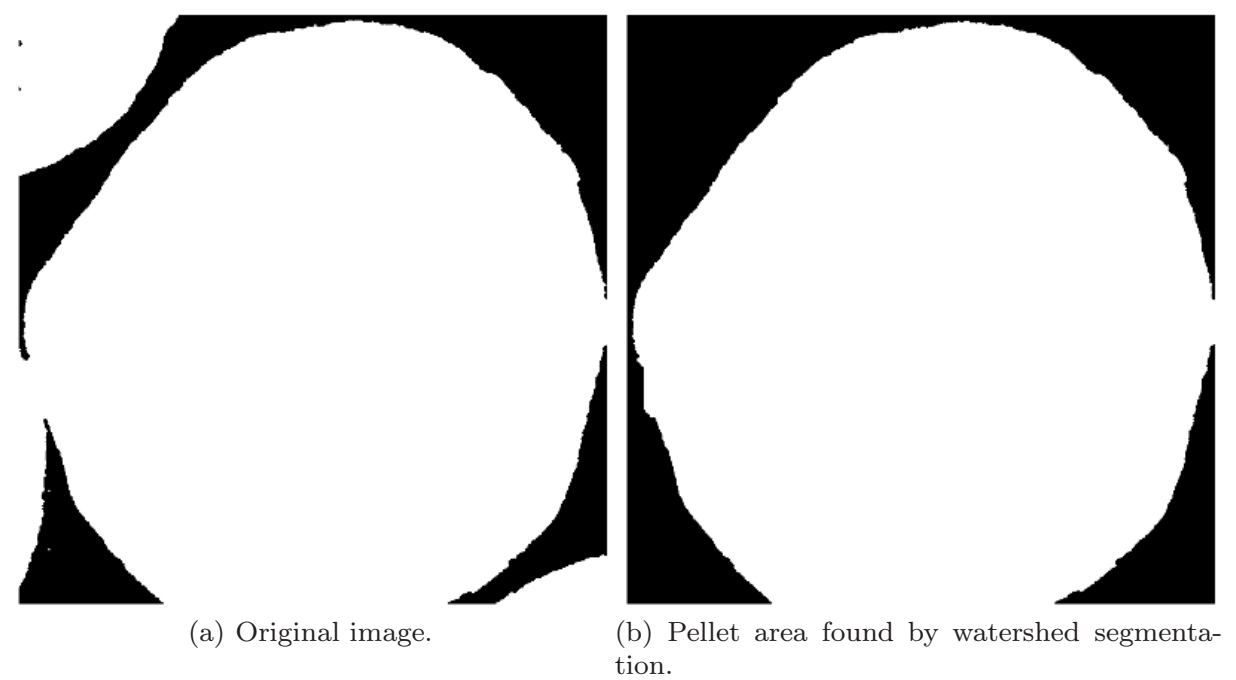

Figure 3: Identified pellet region.

\subsubsection{Individual segmentation}

When determining what is pellet and what is not in individual processing of sub images the corresponding binary area from the binary mosaic (figure 3b is upscaled and used to determine how much of the image that is valid. A sub image and its corresponding valid region is shown in figure 4. To provide a reasonable distinction between pellet perimeter images containing single grains (or images of random stains in the epoxy) and images that contains enough information to be processed, only images containing more than $5 \%$ pellet are considered.

The distance to the pellet perimeter for a given image is an average of the distance from each pixel inside the pellet (in that image) to the nearest point on the pellet perimeter. To convert from number of pixels to micrometers, these values need to be multiplied by 8 to compensate for the downsampling, and then by the pixel calibration value, in this work $0.643 \mu \mathrm{m}$ per pixel at 20X magnification.

Images are individually processed and segmented using thresholds found by Otsu's method (Otsu, 1979). The two required thresholds are found by applying the method twice. The first step is performed to separate the image into foreground and background. This foreground is then thresholded again to separate magnetite and hematite. A step-by-step example of this is shown in figure 5 ]

Global thresholding If the image contains a high proportion of magnetite the presented segmentation technique may fail. If the detected gray- 
Creation of mosaic:

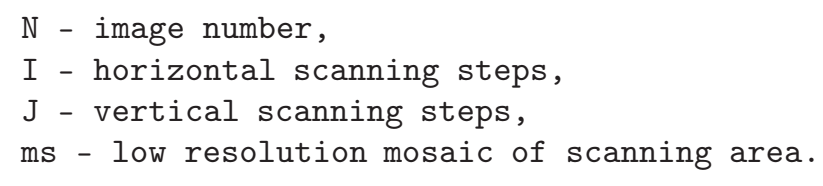

1. row $=\left\lceil\frac{\mathrm{n}}{\mathrm{J}}\right\rceil$

2. $\operatorname{col}=\mathrm{N}-\mathrm{J} \cdot($ row -1$)$

For even rows scanning direction is opposite:

3. if $\mathrm{n} \bmod 2==0$ end $\operatorname{col}=\operatorname{row} \cdot \mathrm{J}-(\mathrm{N}-1)$

4. $\mathrm{ms}=$ empty_matrix $(\mathrm{I}, \mathrm{J})$

5. $\mathrm{ms}($ row, col $)=$ downsample $\left(\mathrm{im}_{\mathrm{N}}, 8\right)$

Thresholding and identification of binary pellet:

$D_{x}$ - structuring element, disk of radius $\mathrm{x}$, to - threshold found by Otsu's method.

6. $\mathrm{msGrad}=\mathrm{ms} \oplus \mathrm{D}_{2}-\mathrm{ms} \ominus \mathrm{D}_{2}$

7. msGrad $\stackrel{\text { thresholding }}{\stackrel{t_{0}}{\longrightarrow}}$ msBin_first

8. msBin_smooth $=$ msBin_first $\bullet D_{8}$

9. msBin_fill = fillHoles(msBin_smooth)

10. $\operatorname{msBin}=\mathrm{msBin}_{-} \mathrm{fill} \ominus \mathrm{D}_{4}$

11. $\operatorname{pelletBin}=$ clearBorder(msBin) (default solution)

Edge clearing if default solution fails, i.e. if pellet connected to mosaic border or neighboring pellet:

pregion - region containing middle pixel of whole mosaic, $\partial$ - rectangular perimeter of whole mosaic, seeds - marker regions for later watershed transform.

12. while (pregion $\cap \partial) \notin \emptyset$ $\mathrm{msBin}=\mathrm{msBin} \ominus \mathrm{D}_{2}$ $\mathrm{msBin}_{\text {regions }}=\mathrm{label}_{(\mathrm{msBin})}$ seeds $=$ seeds $\cup\left\{\right.$ msBin $\left._{\text {regions }}: \operatorname{msBin}_{\text {regions }} \cap p_{\text {region }} \in \emptyset\right\}$ end

13. msBin $^{\mathrm{C}} \stackrel{\text { distancetransform }}{\longmapsto}$ distances

14. regions $=$ watershed(distances, seeds)

15. pelletBin $=\{$ region $: \mathrm{p} \in$ region $\}$ 


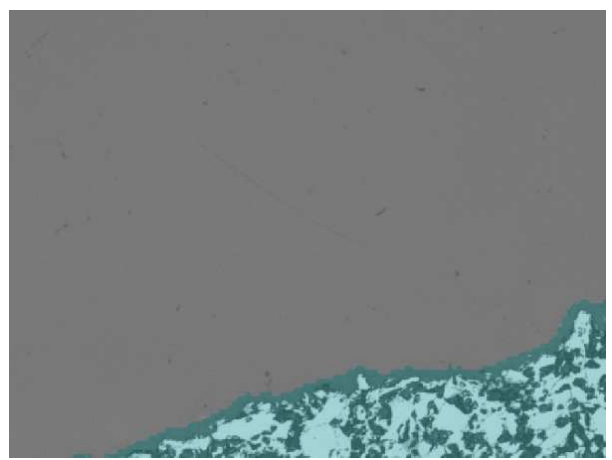

Figure 4: Pellet region marked in turquoise on a pellet perimeter image.

level-threshold between magnetite and hematite for a given image is below the gray-level-value of the magnetite peak in the combined gray-level-histogram of all images of the pellet, we consider that this segmentation failure will have occurred. In this case we use an alternate gray-level-threshold derived by performing Otsu's method on the combined gray-level-histogram of all images, illustrated in figure 8, which shows the combined histogram for all images of one pellet where identified global peaks and thresholds have been marked.

Edges with gray-level equivalent to magnetite Some phenomena (see section 4.2 for discussion) causes the edges of the hematite grains to have the same grayscale value as the magnetite and will therefore be erroneously classified as magnetite. In order to compensate for this effect magnetite located between the outer border of hematite grains and outer border of pores/additives can be added to the hematite and subtracted from the magnetite. The thickness of this edge of pseudo-magnetite is empirically decided to 4 pixels $(2.572 \mu \mathrm{m})$. 


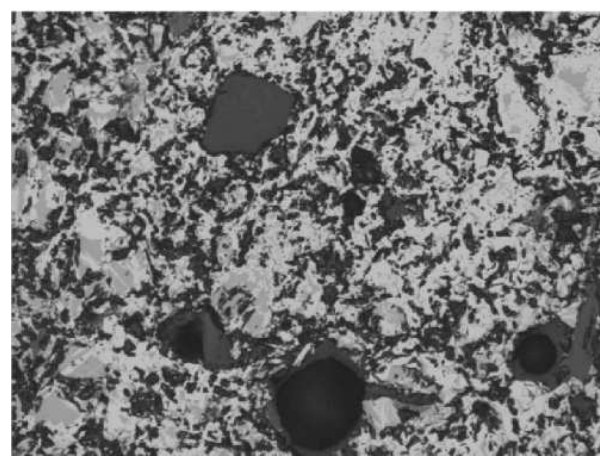

(a) Original image.

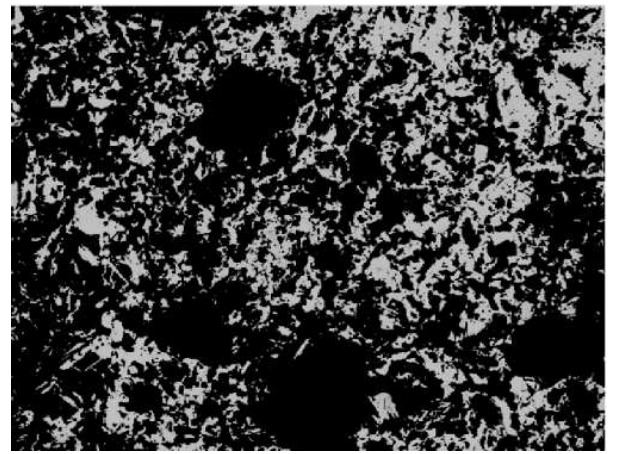

(c) Hematite after second thresholding.

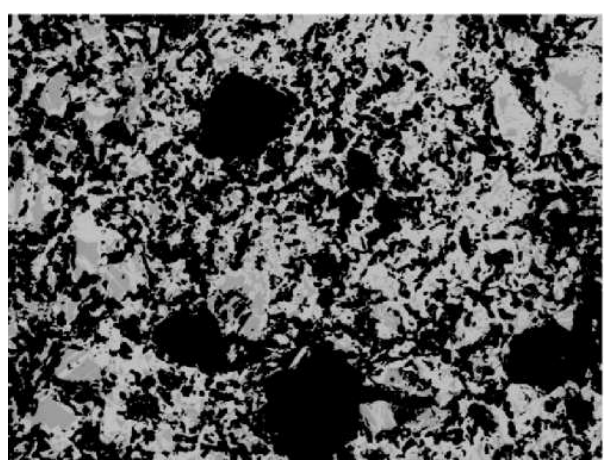

(b) Foreground found by first thresholding.

Figure 5: Step-by-step segmentation.

\section{Results}

\subsection{Image acquisition}

Scanning one pellet results in 300-450 images depending on its size. For each tested pellet a maximum of 5 out of the 350-400 images have been blurry. Both pellets situated in the middle of and closer to the edges of the epoxy sample are possible to scan, even those who create a scanning area that lies outside of the epoxy.

A scan takes somewhere between 2 and 4 hours depending on the amount of epoxy. Images of pure epoxy take a lot longer to retrieve due to focusing issues. The automatic focus can not find a hit on the monotonously gray plastic and therefore has to test all possible values before moving to the next position.

The microscope is placed at a room in the LKAB laboratory illuminated by normal lamps and influenced by some daylight. The procedure has therefore been to retrieve a new reference image before scanning a new pellet. Preliminary test show that this might not always be necessary, but due to time constraints this has not been thoroughly investigated after a sufficient solution had been found. 


\subsection{Image processing}

The total percentages of magnetite, hematite and pores \& additives in each image are calculated and plotted against the distance between the image and the pellet edge. This is done in the same plot for all individual images producing a phase distribution plot like the one in figure 9

The results from each scan are presented with an overview of the segmentation, the histogram with the global thresholds and the phase distribution plot in figures 7, 8 and 9. Also the total phase percentages (area percentages) for the entire pellet are displayed as in table 1

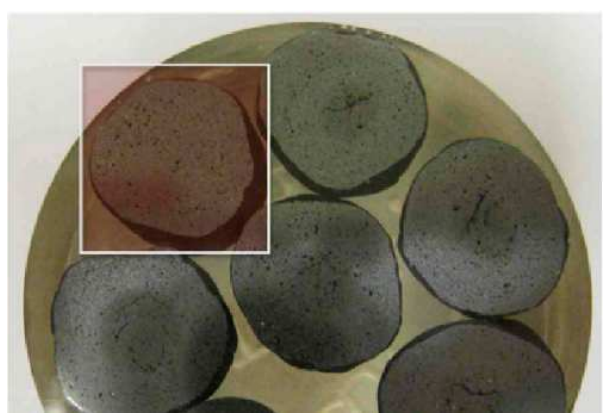

Figure 6: Pellet with scanning area partly outside of epoxy.

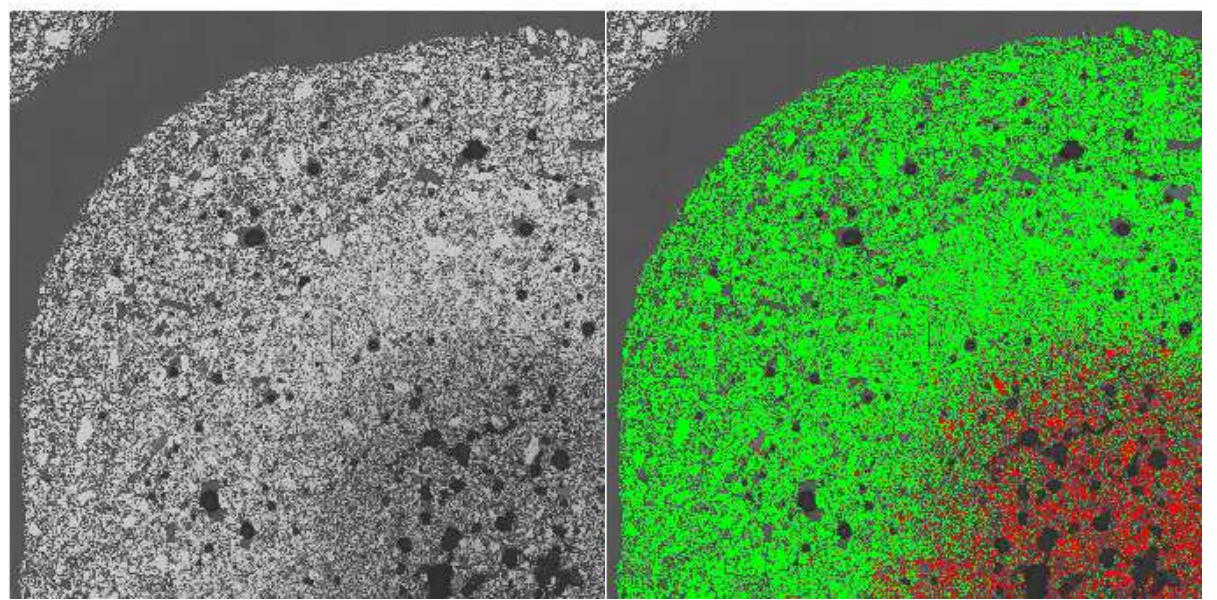

(a) Original pellet.

(b) Results overlaid on original (magnetite red, hematite - green).

Figure 7: Overview mosaic and a mosaic created from the individual segmentation results in the pellet of interest (only $1 / 4$ of the pellet shown). 


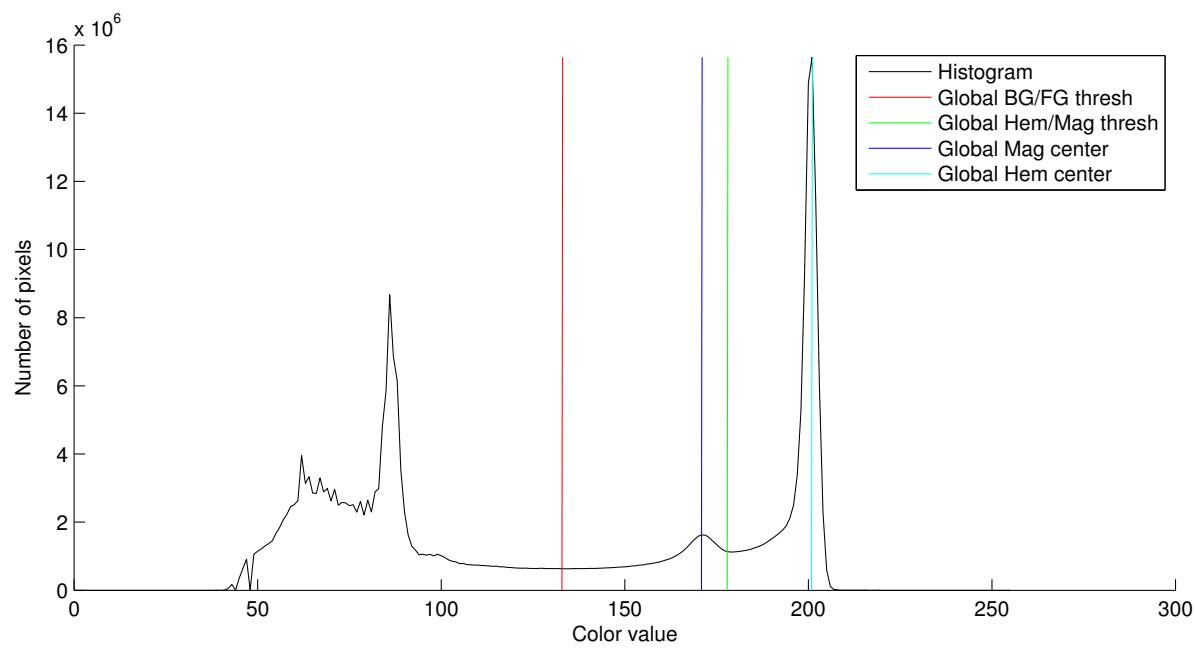

Figure 8: Histogram of all images.

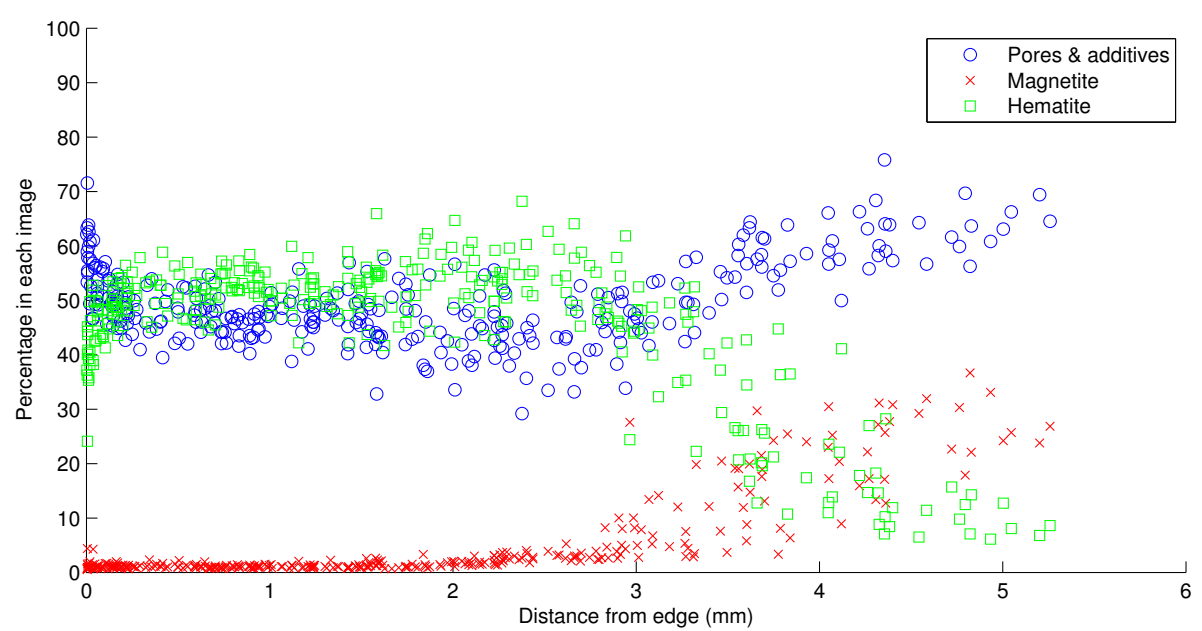

Figure 9: Phase distribution within the pellet. 


\begin{tabular}{l||l}
\multicolumn{2}{c}{ Total phases } \\
\hline \hline Pores: & $48.86 \%$ \\
Magnetite: & $5.23 \%$ \\
Hematite: & $45.91 \%$
\end{tabular}

Table 1: Total phases percentages for the pellet in figure 7

\section{Discussion}

\subsection{Image acquisition}

The blurry images are too few to cause a major problem. They will of course influence the end result due to incorrect segmentation but at a maximum number of 5 out of 400 for one of the studied pellets will give a small bias that can be discarded for the results presented here. An observation is that the blurry images predominantly appear to occur where there are cracks or polishing artifacts in the epoxy so sample preparation is crucial.

\subsection{Image processing}

The automatic segmentation performs well in general. Small problems that occurs for some pellets are most obvious on images with magnetite strokes in the hematite grains as in figure 10. The red overlay shows where the magnetite has been correctly identified but there are some missed strokes in the upper right corner. Out of seven tested pellets the problem is only this obvious in a few images from one of the pellets, in the other cases all individual magnetite regions are found but not completely covered.

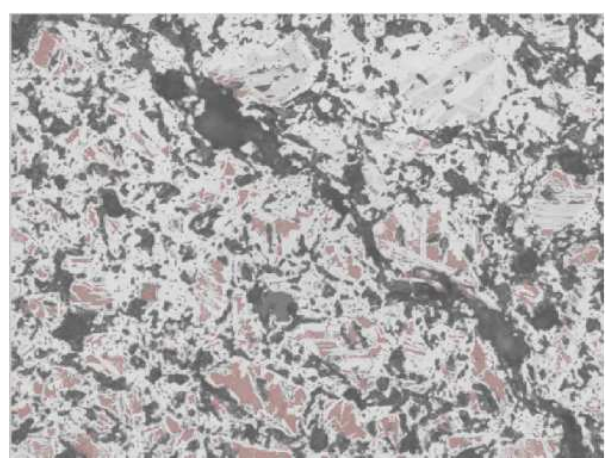

Figure 10: Area where not all magnetite has been found (red overlay is the found magnetite)

Two problems that cannot be solved with manual segmentation techniques are taken care of with the suggested automatic system. The first is the edges of pseudo-magnetite the hematite grains and the second is determining the phase percentages of only the pellet part when analyzing images from the outer perimeter of the pellet, like the one in figure 4.

For individual images, comparing phase percentages with manual segmentations gives similar results, at least when not compensating for the pseudo- 
magnetite (which cannot be done manually). The only phase that we have had knowledge of a ground truth from chemical analysis has been the magnetite, and that number has been used to fine-tune the compensation for the pseudomagnetite.

In order to use this prototype system as a reliable analysis tool the suggestion is to create a large database to get a reference knowledge of what are normal production results. There is also a lot of potential to test and develop this system further as can be seen in the following sections of this chapter.

Edges with gray-level equivalent to magnetite As introduced in section 2.2 .2 edges of pseudo-magnetite occurred around the hematite grains and is falsely identified as magnetite. The cause of this phenomenon is not determined. One thought is that this might originate from the polishing due to the difference in hardness between the hematite grains and the surrounding plastic. Another possible explanation is the effect known as optical smearing mentioned as a possible mentioned in e.g. (Wagner et al., 2008) et al., combined with some blurring.

When doing chemical analysis the normal value of remaining magnetite in a fully oxidized Malmberget pellet lies at $0.8 \%$ (weight fraction). Empirical results show that the performed compensation for the pseudo-magnetite is necessary to get realistic results.

If the edges should be added to pores \& additives or hematite needs to be investigated further. Currently they are added to the latter phase but this is not yet a validated conclusion.

\subsection{Additional development}

A suggestion of the next step is to look deeper into separation of the pores $\&$ additives phase. This might be possible either through analysis of the same type of images that has been used in this work or by putting the samples in fluorescent epoxy which will give a good contrast between additives and plastic. A more thorough investigation out of scope for this work is however needed.

\section{Conclusion}

This paper concludes that a system for automatic microscopy is possible using the suggested methods for image acquisition and analysis.

The image acquisition step uses a modified version of a mosaic routine included in the Leica Qwin Pro software. For the acquisition step a number of problems have been solved. The main problems have been getting all images in focus and compensating for vignetting (uneven illumination).

The image analysis is based on Otsu's thresholding technique from 1979 Otsu (1979). Studies of seven different pellets show that the method successfully segments the images into magnetite, hematite and pores \& additives.

A number of problems were necessary to address in the image analysis step. One example is the pellet region that needs to be found, if the scanned pellet is touching neighboring pellets in the sample only the pellet of interest should be analyzed. Another problem has been that edges of the hematite grains look like magnetite in the images. Both of these problems have been mitigated using morphological image processing techniques.

Using a technique based on this work, engineers could successfully analyze more pellets a day than they do now for the proportion of hematite/magnetite. The biggest advantage is that this could be done in a largely automated man- 
ner whereas today all steps are performed manually. If the image acquisition speed could be further improved, as many as 10-20 pellets could probably be completely analyzed during one day compared to the randomly chosen spots examined today.

\section{References}

Alvarez, J., Wagner, D., Schinazi, G., Gomes, O., De Pinto Maurício, M., Paciornik, S., Viera, M., September 2008. Qualitative and quantitative evaluation of iron ore sinters through digital microscopy. In: Proceedings of the 9th International Congress on Applied Mineralogy. Vol. 1 of CT2008-057-00. pp. $669-672$.

Beucher, S., Meyer, F., 1992. Mathematical Morphology in Image Processing. Marcel Dekker Inc., Ch. 12, pp. 433-481, the Morphological Approach to Segmentation: The Watershed Transform.

Dougherty, E. R., Lotufo, R. A., 2003. Hands-On Morphological Image Processing. Vol. TT59. SPIE - The International Society for Optical Engineering.

Gomes, O., Paciornik, S., 2008. Iron ore quantitative characterisation through reflected light-scanning electron co-site microscope. In: Proceedings of the 9th International Congress on Applied Mineralogy. Vol. 1 of CT2008-059-00. pp. $699-702$.

Lessa, A., Ferreira, H., Abreu, J., Gomes, O., Paciornik, S., September 2007. Automatic classification of hematite types in "pellet feed" through digital image analysis. In: XXI Congresso da Sociedade Brasileira de Microscopia e Microanálise. Vol. 1 of CT2008-057-00.

MATLAB, 2010. version r2009a. URL http://www . mathworks . com

Nellros, F., 2010. Automated image analysis of pellet structure using optical microscopy. Master's thesis, Luleå University of Technology. URL http://pure.ltu.se/portal/files/31174112/LTU-EX-10097-SE.pdf

Niiniskorpi, V., 2004. Development of phases and structures during pelletizing of kiruna magnetite ore. Ph.D. thesis, Åbo Akademi University, report 04-3.

Otsu, N., Jan 1979. A threshold selection method from gray-level histograms. IEEE Transactions on Systems, Man and Cybernetics 9 (1), 62 - 66.

Pirard, E., Lebichot, S., 2004. Image analysis of iron oxides under the optical microscope. In: Proceedings of the 8th International Congress on Applied Mineralogy. Vol. 1 of Applied Mineralogy: Developments in Science and Technology. pp. 153- 156.

Pirard, E., Lebichot, S., Krier, W., 2007. Particle texture analysis using polarized light imaging and grey level intercepts. International Journal of Mineral Processing 84, 299-309.

Ray, F. S., 1994. Applied photographic optics. Focal press (2nd edition), Oxford, England. 
Wagner, D., Roucho, H., Gomes, O., Paciornik, S., Vieira, M., 2008. Iron ore pellet characterization through digital microscopy. In: 2nd International Symposium on Iron Ore. Vol. 1843 of CT2008-064-00. pp. 231 - 236. 